\title{
In Vitro Evaluation of Urinary Stone Comminution with a Clinical Burst Wave Lithotripsy System
}

\author{
Shivani Ramesh,, Tony T. Chen, MD, ${ }^{2}$ Adam D. Maxwell, PhD, ${ }^{1,2}$ Bryan W. Cunitz, MS, ${ }^{1}$ Barbrina Dunmire, MS, \\ Jeff Thiel, BS, RDMS, ${ }^{1}$ James C. Williams, Jr., PhD, ${ }^{3}$ Anthony Gardner, BS, ${ }^{3}$ Ziyue Liu, PhD, ${ }^{4}$ Ian Metzler, MD, \\ Jonathan D. Harper, MD, ${ }^{2}$ Mathew D. Sorensen, MD, MS, FACS, ${ }^{5}$ and Michael R. Bailey, PhD ${ }^{1,2}$
}

\begin{abstract}
Objective: Our goals were to validate stone comminution with an investigational burst wave lithotripsy (BWL) system in patient-relevant conditions and to evaluate the use of ultrasonic propulsion to move a stone or fragments to aid in observing the treatment endpoint.

Materials and Methods: The Propulse-1 system, used in clinical trials of ultrasonic propulsion and upgraded for BWL trials, was used to fragment 46 human stones $(5-7 \mathrm{~mm})$ in either a $15-\mathrm{mm}$ or $4-\mathrm{mm}$ diameter calix phantom in water at either $50 \%$ or $75 \%$ dissolved oxygen level. Stones were paired by size and composition, and exposed to 20-cycle, $390-\mathrm{kHz}$ bursts at 6-MPa peak negative pressure (PNP) and 13-Hz pulse repetition frequency (PRF) or 7-MPa PNP and 6.5-Hz PRF. Stones were exposed in 5-minute increments and sieved, with fragments $>2 \mathrm{~mm}$ weighed and returned for additional treatment. Effectiveness for pairs of conditions was compared statistically within a framework of survival data analysis for interval censored data. Three reviewers blinded to the experimental conditions scored ultrasound imaging videos for degree of fragmentation based on stone response to ultrasonic propulsion.

Results: Overall, 89\% (41/46) and 70\% (32/46) of human stones were fully comminuted within 30 and 10 minutes, respectively. Fragments remained after 30 minutes in $4 \%(1 / 28)$ of calcium oxalate monohydrate stones and $40 \%$ (4/10) of brushite stones. There were no statistically significant differences in comminution time between the two output settings $(p=0.44)$, the two dissolved oxygen levels $(p=0.65)$, or the two calyx diameters $(p=0.58)$. Inter-rater correlation on endpoint detection was substantial (Fleiss' kappa $=0.638, p<0.0001)$, with individual reviewer sensitivities of $95 \%, 86 \%$, and $100 \%$.

Conclusions: Eighty-nine percent of human stones were comminuted with a clinical BWL system within 30 minutes under conditions intended to reflect conditions in vivo. The results demonstrate the advantage of using ultrasonic propulsion to disperse fragments when making a visual determination of breakage endpoint from the real-time ultrasound image.
\end{abstract}

Keywords: lithotripsy, ultrasound, kidney stones

\section{Introduction}

O UR TEAM IS developing a system to image, fragment, and reposition urinary stones that can be used in an office setting with minimal risk to the subject. ${ }^{1}$ Such a system integrates three ultrasound-based technologies working together. First, imaging of stones is carried out using S-mode ultrasound, a method that combines power Doppler and B-Mode imaging to more sensitively and specifically detect stones by Doppler twinkling. ${ }^{2}$ Second, fragmentation of stones is carried out using burst wave lithotripsy (BWL), which applies focused, cyclic ultrasound pulses to a stone at pressure amplitudes lower than with extracorporeal shockwave lithotripsy (SWL). ${ }^{3,4}$ BWL is applied transcutaneously from a handheld transducer applied to the skin. The transducer includes the annular therapy element, which surrounds the independent imaging probe that provides treatment guidance. ${ }^{1}$ The same therapy element and transducer are also

\footnotetext{
${ }^{1}$ Applied Physics Laboratory, Center for Industrial and Medical Ultrasound, University of Washington, Seattle, Washington, USA.

${ }^{2}$ Department of Urology, University of Washington School of Medicine, Seattle, Washington, USA.

Departments of ${ }^{3}$ Anatomy, Cell Biology and Physiology and ${ }^{4}$ Biostatistics, Indiana University School of Medicine, Indianapolis, Indiana, USA.

${ }^{5}$ Division of Urology, VA Puget Sound Health Care System, Seattle, Washington, USA.
} 
used to perform ultrasonic propulsion, which generates acoustic radiation force on stones or fragments to propel them and promote their clearance. ${ }^{5-7}$ Each of these three technologies has undergone testing of safety and effectiveness on the bench and in animals. 1

In preparation for a human trial, we sought to compare comminution effectiveness of BWL at two treatment settings across a range of variables (stone composition, dissolved gas level, and tissue confinement) anticipated to be encountered in human use. Each of these variables has been shown to impact BWL and SWL effectiveness in vitro. ${ }^{3,8,9}$ Although the composition of a stone is not likely to be known a priori in clinical practice, it is important to understand how BWL comminution effectiveness varies across common stone types. The dissolved oxygen level impacts the potential for cavitation and its activity. ${ }^{10}$ As with SWL, BWL must avoid cavitation clouds to be effective and prevent tissue injury. ${ }^{11,12}$ Cavitation clouds can shield the acoustic energy from reaching the stone and damaged tissue. Although the dissolved gas concentration in urine has been measured to be $70 \%,{ }^{13}$ the composition of gases is different from that dissolved in water in vitro, thus it is difficult to mimic in vivo conditions. Previous results have also shown that the amount of fluid space surrounding a stone impacts comminution effectiveness in SWL and BWL, and it has been proposed that confined clouds of debris harbor cavitation nuclei that increase the likelihood of a cavitation cloud. ${ }^{8,14}$

The lack of imaging feedback to determine treatment effect can limit SWL's clinical application. By comparison, in
BWL, we aim to combine B-Mode imaging and ultrasonic propulsion. Whereas it can be difficult to discriminate an intact stone from a collection of stone fragments at rest, regardless of mode of imaging, it has been shown that ultrasonic propulsion can be used to create differential motion helpful in observing that fragmentation has occurred. ${ }^{5}$ By observing the stone response on B-Mode to ultrasonic propulsion, we hypothesize that the degree of fragmentation can be determined to decide if the treatment endpoint has been achieved.

\section{Materials and Methods}

In this article we tested the investigational system with four common stone types, two oxygen saturation levels, and two levels of tissue confinement that encompass different conditions potentially encountered in humans.

The experimental configuration is given in Figure 1. Stones were fragmented within a tissue phantom intended to mimic the conditions in the kidney or ureter. ${ }^{9,14}$ The therapy transducer was affixed pointing down in a 190-L tank on a 3 -axis positioner. The tank was filled with deionized water filtered through a $0.5-\mu \mathrm{m}$ sediment filter. The water was held at $18^{\circ} \mathrm{C}$ and degassed to maintain a dissolved oxygen level of $50 \%$ (previously shown to simulate the BWL cavitation threshold in vivo $)^{9}$ or a worse-case condition of $75 \%$ of the saturation level (Oxi 330i meter with a CellOx 325 probe; WTW, Weilheim, Germany). Stones were held in a $60-\mathrm{mm}$ diameter by $70-\mathrm{mm}$ tall cylindrical tissue phantom made of either polyvinyl chloride (PVC) (Soft Baits; Do-it Molds, Denver,
FIG. 1. Experimental arrangement. The stone was enclosed in a water-filled cavity within a tissue mimicking phantom submerged in a water bath. The BWL and ultrasonic propulsion ultrasound bursts were applied from a transducer positioned above the phantom. The realtime ultrasound image of the transducer was recorded to video. The " $\mathrm{x}$ " indicates the focus on the transducer. $\mathrm{BWL}=$ burst wave lithotripsy.

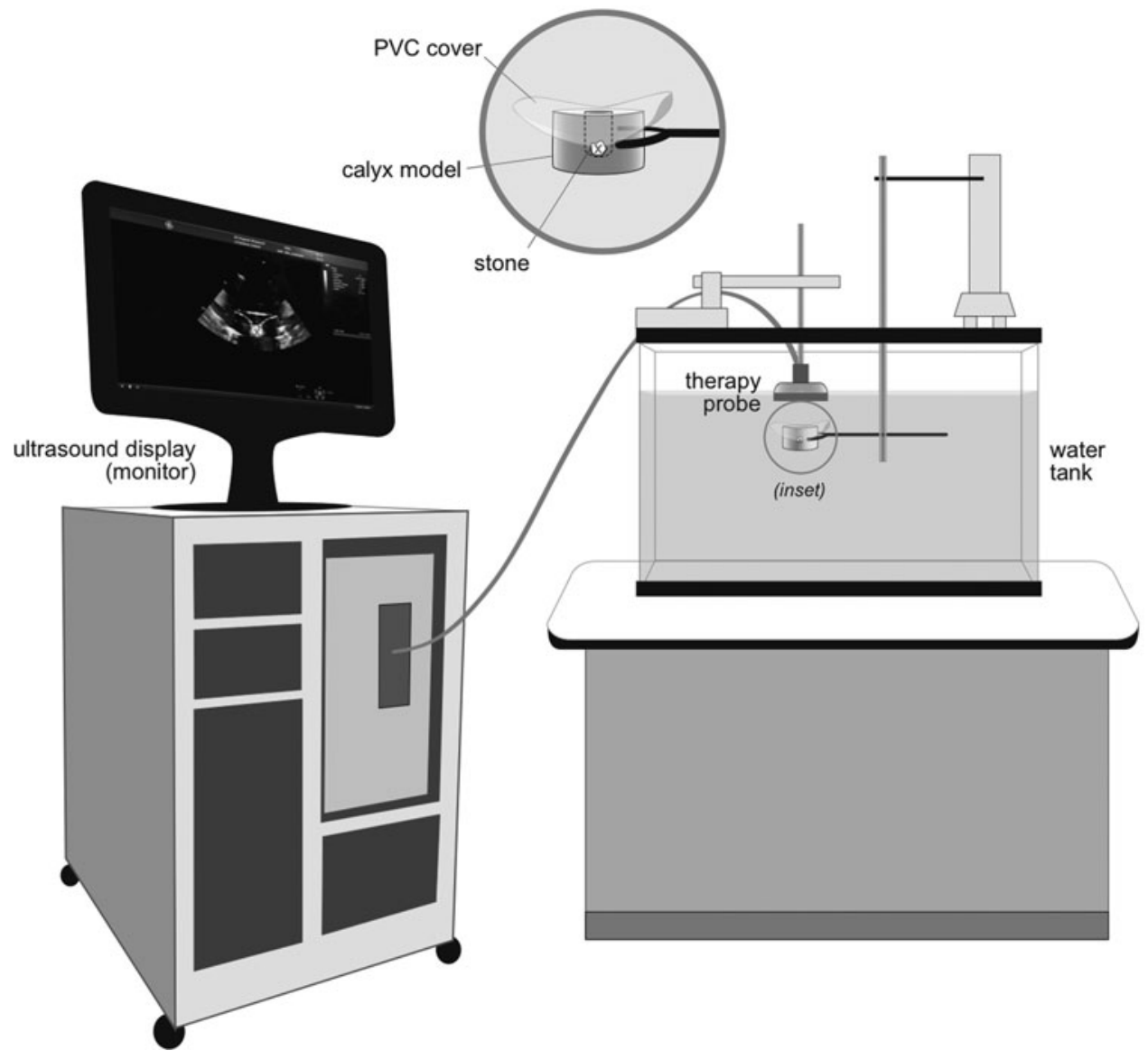


IA) or ballistic gel (Clear Ballistics, Greenville, SC). ${ }^{14}$ The phantom contained a depression or cavity in the top surface. The depression dimensions were $15-\mathrm{mm}$ diameter by $40-\mathrm{mm}$ depth used previously to represent a kidney calyx ${ }^{14}$ or 4-mm diameter by $6-\mathrm{mm}$ depth to represent the ureter. A 3-mm thick disk of PVC was used to cover both cups for all experiments.

Stones were broken with the Propulse 1 system configured for BWL outputs as described in Bailey et al. ${ }^{1}$ As designed for clinical use, the operator places the probe on the skin, views the stone with ultrasound imaging, and then applies the therapy by a footswitch without interrupting real-time ultrasound imaging. The focal geometry was measured from a full-field scan conducted at low pressure using a piezoelectric hydrophone (Model HGL-200; Onda Corp., Sunnyvale, CA) and confirmed through simulations (Field II). ${ }^{15,16}$ The focal beamwidth was $5 \mathrm{~mm}$ at the narrowest. The peak free-field focal pressure was measured using a fiber optic hydrophone (Model FOPH 2000; RP Acoustics, Leutenbach, Germany). Each pulse consisted of 20 cycles at a center frequency of $390 \mathrm{kHz}$. The pulses were delivered at $6 \mathrm{MPa}$ peak negative pressure (PNP) and $13 \mathrm{~Hz}$ pulse repetition frequency (PRF) or $7 \mathrm{MPa}$ PNP and $6.5 \mathrm{~Hz}$ PRF.

Forty-six human stones (63-358 mg, 5-7 mm; Beck Labs, Indianapolis, IN) were used. The history of the stones is not known. Primary composition was determined by micro-CT and spectroscopy, ${ }^{17}$ and resulted in 28 calcium oxalate monohydrate (COM), 10 brushite, 6 struvite, and 2 apatite. Stones were divided into paired groups based on size and composition. In $87 \%$ (20 of 23) of the pairs, both stones came from the same subject. All stones were soaked in deionized water for at least 1 week before the experiment. ${ }^{18}$

A stone was placed into the tissue phantom. The transducer was moved such that the stone was positioned at the crosshairs on the ultrasound image, which represented the position of peak focal pressure of the therapy beam. The recording of the ultrasound video was initiated and the stone was exposed to a fixed BWL setting for 5 minutes. During exposure, an audible clicking was heard at the BWL PRF. Empirically, the sound appears to be loudest when the acoustic beam is targeted on the stone. Thus, in addition to using ultrasound imaging to target the stone, the position of the transducer was continuously adjusted over a range of $\sim 3 \mathrm{~mm}$ to maintain a clear image of the stone and maximize the audible clicking feedback, similar to how we expect to use the handheld probe in human trials.
After 5 minutes of treatment with BWL, an ultrasonic propulsion burst was applied to move the stone or fragments and the phantom was removed from the water bath. The stone fragments were passed over a 2-mm sieve, and the remaining fragments $(>2 \mathrm{~mm}$ in size) weighed. The remaining fragments were then placed back in the holder, and treatment resumed. Exposures were repeated in 5-minute intervals until there were no remaining fragments $>2 \mathrm{~mm}$ (complete comminution) or 40 minutes.

Data are reported as ratio of the mass of fragments $>2 \mathrm{~mm}$ to the original stone mass as a function of time for each of the test conditions. SAS PROC ICLIFETEST, ICPHREG, and R package IRR were used for statistical analysis. Statistical analysis of the fragmentation effectiveness was performed under the framework of a survival data analysis for interval censored data, because time-to-complete comminution was censored at 5-minute intervals. The effects of each individual factor were first summarized by median time to comminution and then compared among levels using a generalized logrank test. Their overall effects were then jointly evaluated using a multivariable proportional hazard model with factors as setting ( $6 \mathrm{MPa}$ and $13 \mathrm{~Hz}$ or $7 \mathrm{MPa}$ and $6.5 \mathrm{~Hz}$ ), container (large $v s$ small), gas content (50\% or $75 \%$ ), and type of stone (artificial stones excluded).

Video segments of each propulsion push were collected to evaluate if stone fragmentation could be determined by fragment response during ultrasonic propulsion. Three reviewers blinded to the experimental conditions categorized each video as (1) still intact, (2) fragmented $<50 \%$, (3) fragmented $>50 \%$, or (4) fully comminuted. Categories 1 and 2 were then combined to represent stones necessitating more treatment, and Categories 3 and 4 were combined to describe stones that did not require further treatment for a binary statistical comparison. Reviewer results were compared with known results from the sieving. Fleiss' Kappa was calculated to quantify the inter-rater agreement. The sensitivity, specificity, positive predictive value, and negative predictive value of visual observations compared with measured outcomes were calculated for each individual rater.

\section{Results}

Figure 2 provides the comparison of the comminution effectiveness of the two BWL output settings for all human

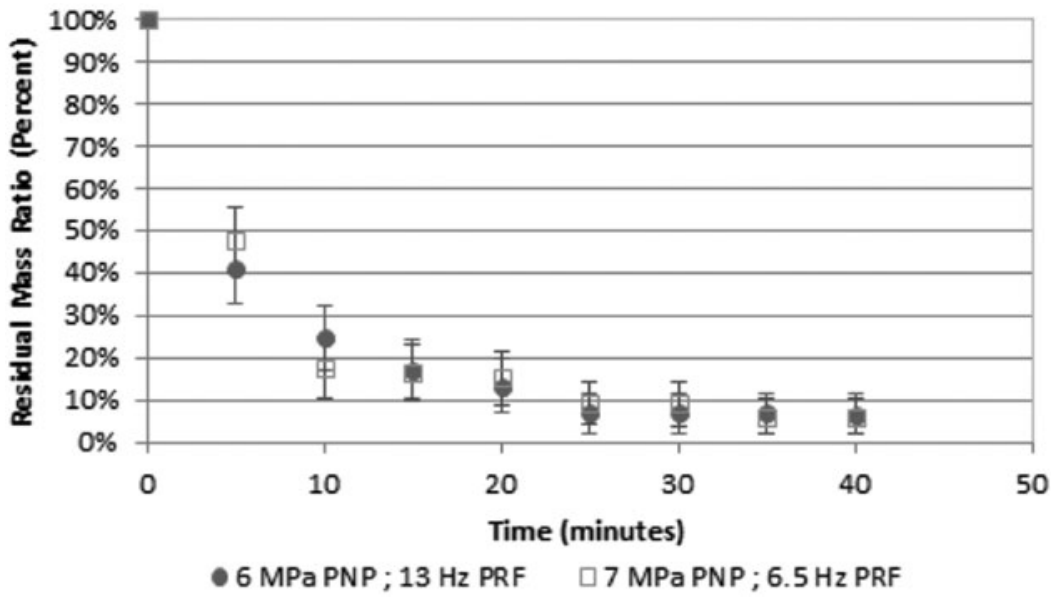

FIG. 2. Comminution effectiveness of the two BWL output settings. On average the remaining stone mass $>2 \mathrm{~mm}$ fell from $100 \%$ at the start to $21 \%$ at 10 minutes and $8 \%$ after 30 minutes. Seventy percent of stones were fully comminuted within 10 minutes, and $89 \%$ of stones were fully comminuted with 30 minutes. There was no statistical difference between the two settings $(p=0.44)$; both fragmented the stones effectively. The error bars represent the standard error. $n=23$ for each setting. 
FIG. 3. BWL comminution effectiveness across different stone compositions. The majority of human stones, including apatite $(n=2)$, struvite $(n=6)$, and COM $(n=28)$, were fully comminuted within 10 minutes. Four of 10 brushite stones were more resistant to comminution. $\mathrm{COM}=$ calcium oxalate monohydrate.

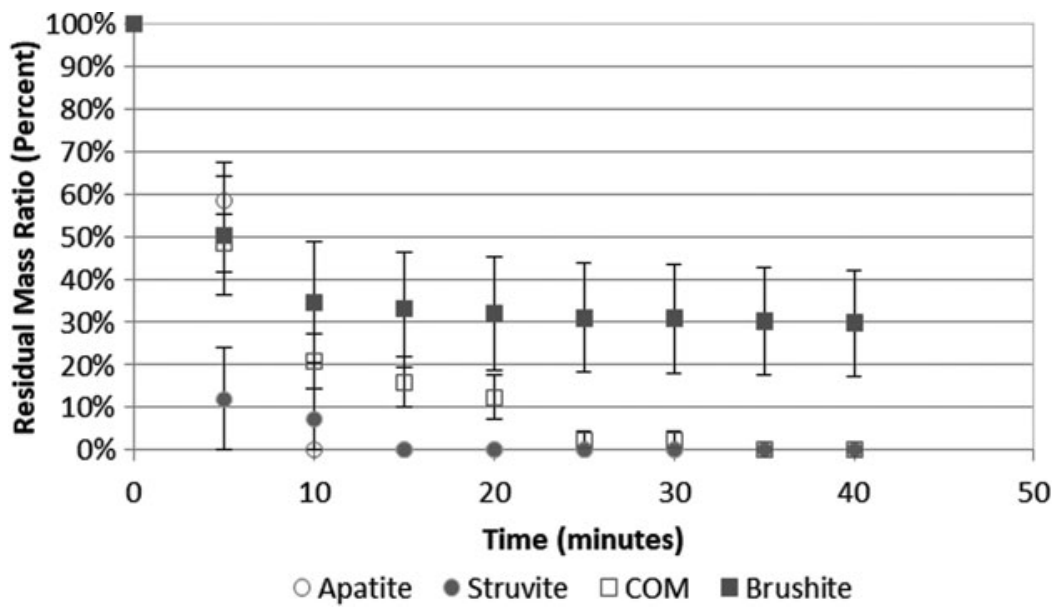

Figure 6 shows representative ultrasound images of an intact stone and fragments of a fully comminuted stone when exposed to ultrasonic propulsion. Inter-rater correlation in identifying whether or not a stone necessitating more treatment was "substantial," the second highest category (Fleiss' kappa $=0.638$ with $p<0.0001) .{ }^{19}$ Table 1 provides the sensitivity, specificity, positive predictive value, and negative predictive value for the three reviewers $\mathrm{A}, \mathrm{B}$, and $\mathrm{C}$. There were five cases of 50 where two or more reviewers differed from the true measurement. In only one case did the reviewers underestimate the degree of comminution. In four cases where the stone underwent significant fragmentation, but breakage not greater than our threshold of 50\%, the reviewers overestimated the degree of fragmentation. The reviewers could not statistically differentiate between $100 \%$ and $50 \%$ comminuted with the conditions and numbers, but in all the $50 \%$ cases, the stone was broken to at least four fragments with no fragments larger than $3 \mathrm{~mm}$.

\section{Discussion}

A system for clinical trials of BWL was tested in a phantom model and shown to effectively comminute four types of human stones with $70 \%$ fully comminuted (all fragments $<2 \mathrm{~mm}$ ) within 10 minutes and $89 \%$ fully comminuted within
FIG. 4. BWL stone comminution effectiveness $v s$ time for two different dissolved oxygen levels. No statistical difference was seen between the two different dissolved oxygen levels $(p=0.65)$. This comparison was conducted with brushite stones. The results are not changed when the two COM stones broken in the high gas content are included.

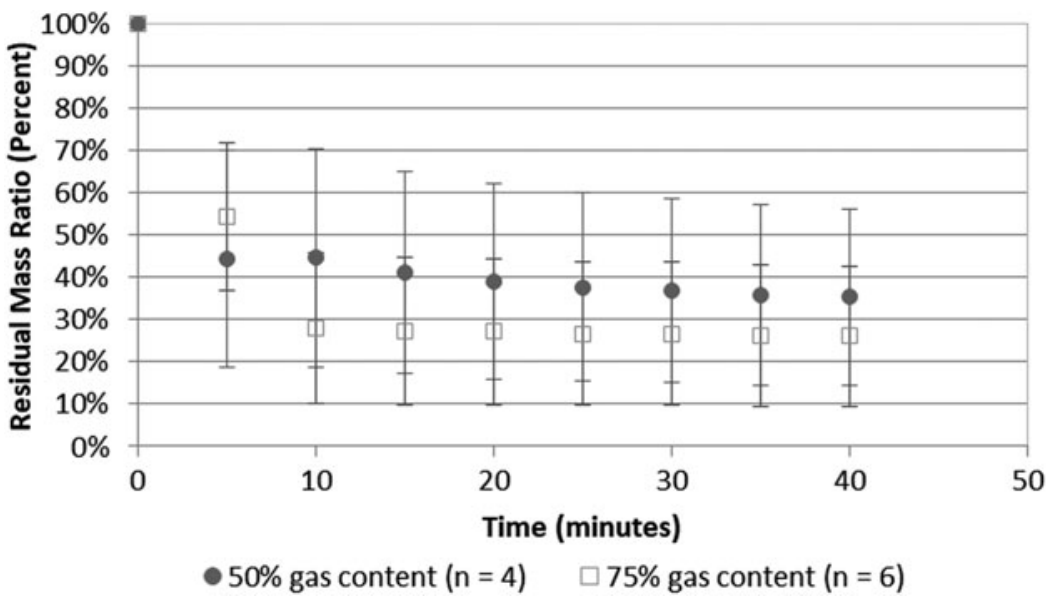




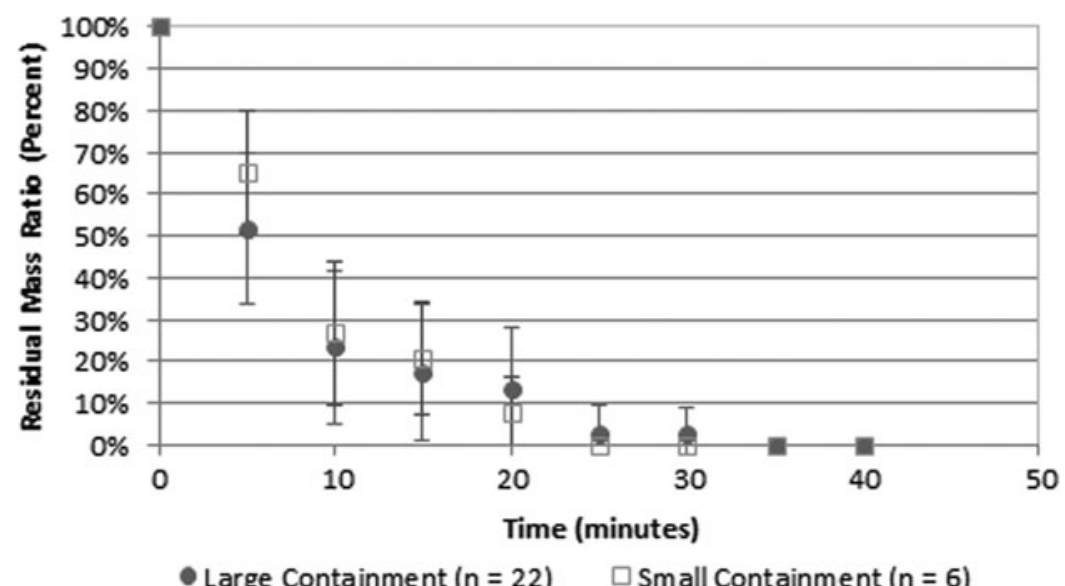

FIG. 5. BWL stone comminution effectiveness vs time for two different containment (fluid) volumes. No statistical difference was seen between the two different containment volumes $(p=0.58)$. This comparison was conducted with COM stones.
30 minutes. Fragments $>2 \mathrm{~mm}$ remained after 40 minutes only in four brushite stones, a composition known to be difficult to comminute with SWL. ${ }^{20}$

The results showed BWL was equally effective across two treatment parameters settings: $6 \mathrm{MPa}$ and $13 \mathrm{~Hz}$ and $7 \mathrm{MPa}$ and $6.5 \mathrm{~Hz}$. Ideally, pressure and PRF should be maximized to break stones as quickly as possible. But both parameters can impact the formation of a cavitation cloud, which reduces energy delivered to the stone and results in tissue injury. ${ }^{21}$ Likewise tight "tissue" confinement has been hypothesized to constrain helpful cavitation at the stone surface when artificial stones broke slower with BWL in a ureter model than in a water bath, ${ }^{8}$ and similarly, having a smaller fluid space confines dust that can promote a cavitation cloud and reduced SWL comminution of artificial model stones. ${ }^{14}$ Finally, the level of dissolved gas in vivo is difficult to mimic in vitro, and a higher level also creates more risk of a cavitation cloud and then reduce BWL effectiveness. ${ }^{9}$ However, for the parameter space investigated here meant to replicate the range of in vivo conditions, both output settings are conservative as they were designed not to produce a cavitation cloud and were shown not to have reduced effectiveness at the highest dissolved oxygen level or smallest fluid volume on interest. This was confirmed in that neither output generated a cavitation cloud

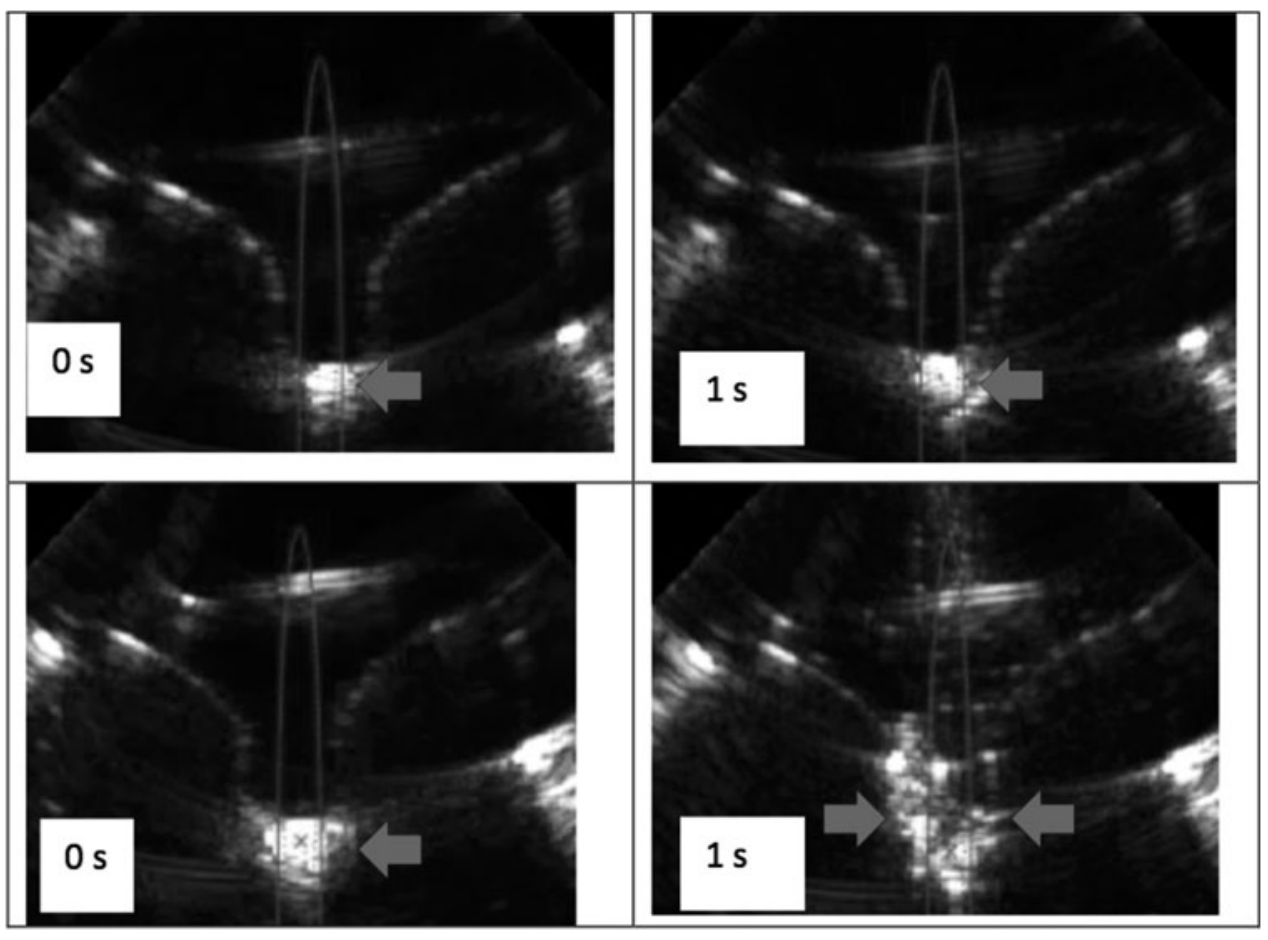

FIG. 6. Ultrasound images captured during ultrasonic propulsion of a minimally broken stone (upper frames) and a completely fragmented stone (lower frames). Both show frames captured from a video of stone motion during a single 3 -second ultrasonic propulsion burst. The arrow points to the moving stone and/or fragments. The left frames are from before the ultrasonic propulsion application. Before propulsion, it is difficult to differentiate an unbroken stone from a pile of fragments. The right frames correspond to 1 second into the ultrasonic propulsion burst. Arrows point to echogenic stone or fragments. In the upper sequence, all three reviewers reported one large fragment. In the lower sequence, all three reviewers reported a collection of fragments all $<2 \mathrm{~mm}$. The reviewers viewed the entire movie not just the still images. 
Table 1. Sensitivity, Specificity, Positive

Predictive Value, and Negative Predictive VALUe FOR THE THREe REVIEWERS

\begin{tabular}{lcccc}
\hline Reviewer & $\begin{array}{c}\text { Sensitivity } \\
(\%)\end{array}$ & $\begin{array}{c}\text { Specificity } \\
(\%)\end{array}$ & $\begin{array}{c}\text { Positive } \\
\text { predictive } \\
\text { value (\%) }\end{array}$ & $\begin{array}{c}\text { Negative } \\
\text { predictive } \\
\text { value (\%) }\end{array}$ \\
\hline A & 95.2 & 66.7 & 74.1 & 93.3 \\
B & 85.7 & 71.4 & 75.0 & 83.3 \\
C & 100 & 85.7 & 87.5 & 100 \\
\hline
\end{tabular}

detectable by real-time ultrasound imaging that would direct the user to dial back the exposure. . $^{2,23}$

In addition, we presented evidence of a new technique to determine the endpoint of treatment, that is, when the stone has been comminuted, which can be unclear in SWL. ${ }^{24,25}$ This technique involves real-time ultrasound monitoring while repositioning the stone or fragments with ultrasonic propulsion. The results presented here were consistent with observations in the first human trial of ultrasonic propulsion and hold promise; although further work is needed to develop technique for clinical utility. ${ }^{5}$ In addition, it has previously been shown that interleaving propulsion pulses with BWL accelerates comminution potentially by removing shielding by fragments or bubbles. ${ }^{26}$

As an initial target, we focused on moderate-sized stones or residual fragments, 5-7 mm based on the beamwidth of our probe. BWL, like SWL, is expected to be most effective if the stones and beamwidth are comparable size. ${ }^{27,28}$ Studies have shown there is no technical obstacle to designing a larger beam to treat larger stones. ${ }^{3}$ Broad focus BWL transducers have been shown to break large artificial stones faster. ${ }^{29}$

\section{Conclusions}

A clinical BWL system comminuted $70 \%$ of human stones completely within 10 minutes under a range of conditions mimicking those in a human patient. The degree of comminution was effectively determined by applying ultrasonic propulsion to disperse the stone fragments under real-time ultrasound guidance. Together these results indicate current BWL parameters are robust and may be made more efficient and maintained safe by increasing output to a level just below where a cavitation cloud is detected. Such results are encouraging at the advent of the first clinical trials of BWL.

\section{Acknowledgments}

The authors thank Christopher Hunter and Wayne Kreider, $\mathrm{PhD}$ at the Center for Industrial and Medical Ultrasound for their help in setting up the experiment. The authors also thank Charles Robling at Indiana University for measuring the stone composition. Furthermore, this material is also the result of work supported by resources from the Veterans Affairs Puget Sound Health Care System, Seattle, Washington.

\section{Author Disclosure Statement}

Maxwell, Cunitz, Dunmire, Bailey, and Sorensen have consulting agreements with and equity in SonoMotion, Inc., which has licensed this technology from the University of Washington.

\section{Funding Information}

The work was supported by National Institute of Diabetes and Digestive and Kidney Diseases (NIDDK) P01-DK043881 and K01-DK104854.

\section{References}

1. Bailey MR, Wang YN, Kreider W, et al. Update on clinical trials results of kidney stone repositioning and preclinical results of stone breaking with one ultrasound system. Proc Meet Acoust 2018;35:020004.

2. Cunitz BW, Dunmire B, Sorensen MD, et al. Quantification of renal stone contrast with ultrasound in human subjects. J Endourol 2017;31:1123-1130.

3. Maxwell AD, Cunitz BW, Kreider W, et al. Fragmentation of renal calculi in vitro by focused ultrasound bursts. J Urol 2015;193:338-344.

4. Maxwell AD, Wang YN, Kreider W, et al. Evaluation of renal stone comminution and injury by burst wave lithotripsy in a pig model. J Endourol 2019;33:787-792.

5. Harper JD, Cunitz BW, Dunmire B, et al. First-in-human clinical trial of ultrasonic propulsion of kidney stones. J Urol 2016;195 (4 Part 1):956-964.

6. Harper JD, Sorensen MD, Cunitz BW, et al. Focused ultrasound to expel calculi from the kidney: Safety and efficacy of a clinical prototype device. J Urol 2013;190: 1090-1095.

7. Janssen KM, Brand TC, Cunitz BW, et al. Safety and effectiveness of a longer focal beam and burst duration in ultrasonic propulsion for repositioning urinary stones and fragments. J Endourol 2017;31:793-799.

8. Ahn J, Kreider W, Hunter C, et al. Improving environmental and stone factors toward a more realistic in vitro lithotripsy model. J Acoust Soc Am 2017;141:3673-3674.

9. Hunter C, Cunitz B, Dunmire B, et al. Impact of stone characteristics on cavitation in burst wave lithotripsy. Proc Meet Acoust 2018;35:020005.

10. Pishchalnikov YA, Zancanaro AJ, Williams JC, et al. Gas content of the medium surrounding a stone has a significant effect on the efficiency of stone breakage in shock wave lithotripsy. J Acoust Soc Am 201;127:1761.

11. Pishchalnikov YA, McAteer JA, Williams JC, Jr., Pishchalnikova IV, Vonderhaar RJ. Why stones break better at slow shockwave rates than at fast rates: In vitro study with a research electrohydraulic lithotripter. J. Endourol 2006; 20:537-541.

12. Pishchalnikov YA, McAteer JA, Bailey MR, et al. Bubble proliferation in shock wave lithotripsy. J Acoust Soc Am 2007;121:3081.

13. Hwang EY, Fowlkes JB, Carson PL. Variables controlling contrast generation in a urinary bladder model. J Acoust Soc Am 1998;103:3706-3716.

14. Randad A, Ahn J, Kreider W, et al. The effect of dust on fragmentation of kidney stones by shock wave lithotripsy in tissue phantoms. J Endourol 2019;33:400-406.

15. Jensen JA. Field: A Program for Simulating Ultrasound Systems, Paper presented at the 10th Nordic-Baltic Conference on Biomedical Imaging Published in Medical \& Biological Engineering \& Computing, Tampere, Finland, 1996;34:351-353.

16. Jensen JA, Svendsen NB. Calculation of pressure fields from arbitrarily shaped, apodized, and excited ultrasound transducers, IEEE Trans Ultrason Ferroelectr Freq Control 1992;39:262-267. 
17. Williams JC, McAteer JA, Evan AP, et al. Micro-computed tomography for analysis of urinary calculi. Urol Res 2010; 38:477-484.

18. McAteer JA, Williams JC, Cleveland RO, et al. Ultracal-30 gypsum artificial stones for research on the mechanisms of stone breakage in shock wave lithotripsy. Urol Res 2005; 33:429-434.

19. Landis JR, Koch GG. The measurement of observer agreement for categorical data. Biometrics 1977;33:159-174.

20. Williams JC, Saw KC, Paterson RF, et al. Variability of renal stone fragility in shock wave lithotripsy. Urology 2003;61:1092-1096.

21. Sapozhnikov OA, Khokhlova VA, Bailey MR, et al. Effect of overpressure and pulse repetition frequency on shock wave lithotripsy. J Acoust Soc Am 2002;112:1183-1195.

22. May PC, Kreider W, Maxwell AD, et al. Detection and evaluation of renal injury in burst wave lithotripsy using ultrasound and magnetic resonance imaging. J Endourol 2017;31:786-792.

23. Maeda K, Colonius T, Maxwell AD, et al. Modeling and numerical simulation of the bubble cloud dynamics in an ultrasound field for burst wave lithotripsy. Proc Meet Acoust 2018;35:020006.

24. Owen NR, Bailey MR, Crum LA, et al. The use of resonant scattering to identify stone fracture in shock wave lithotripsy. J Acoust Soc Am 2007;121:EL41.

25. Leighton TG, Fedele F, Coleman AJ, et al. A passive acoustic device for real-time monitoring of the efficacy of shockwave lithotripsy treatment. Ultrasound Med Biol 2008;34:1651-1665.

26. Zwaschka TA, Ahn JS, Cunitz B, et al. Combined burst wave lithotripsy and ultrasonic propulsion for improved stone fragmentation. J Endourol 2018;32:344-349.

27. Eisenmenger $W$. The mechanisms of stone fragmentation in ESWL. Ultrasound Med Biol 2001;27:683-693.
28. McAteer JA, Williams JC, Bailey MR, et al. Strategies for improved shock wave lithotripsy. Minerva Urol Nefrol 2005;57:271.

29. Randad A, Ghanem MA, Bailey MR, et al. Design of a transducer for fragmenting large kidney stones using burst wave lithotripsy. J Acoust Soc Am 2020. Proceedings of meetings on acoustics Acoustical Society of America 35(1): 020007. DOI: 10.1121/2.0000954.

Address correspondence to:
Michael R. Bailey
Applied Physics Laboratory
Center for Industrial and Medical Ultrasound
University of Washington
1013 NE 40th Street
Seattle, WA 98105

USA

E-mail: bailey@apl.washington.edu

DOI: 10.1089/end.2020.0358

\title{
Editorial Comment on: "In Vitro Evaluation of Urinary Stone Comminution with a Clinical Burst Wave Lithotripsy System" by Ramesh et al.
}

\author{
Pei Zhong, PhD
}

$\mathbf{T}$ He University of Washington team deserves to be congratulated for developing burst wave lithotripsy (BWL) technology within 5 years from concept, through in vitro ${ }^{1}$ and in vivo ${ }^{2}$ studies, to a complete system ready for clinical trials. BWL utilizes pressure waves of $390 \mathrm{kHz}$ frequency in 20-cycle bursts to crumble renal calculi by progressively opening up microcracks in the stone material. Compared with extracorpo- real shockwave lithotripsy (SWL), BWL may provide an officebased modality for stone management solely driven by ultrasonic (US) technologies to image, fragment, and reposition stones in the kidney. In this in vitro study, ${ }^{3}$ the authors confirm that 5-7 mm kidney stones of various compositions can be comminuted within 30 minutes in tissue phantoms under clinically relevant test environments. Moreover, the potential of US

Department of Mechanical Engineering and Materials Science, Duke University, Durham, North Carolina, USA. 